\title{
Time frequency analysis of ultrasound Doppler signal by S-transform and Wigner-Ville distribution
}

\author{
M. Latfaoui ${ }^{1}$, F. Bereksi Reguig ${ }^{2}$ \\ ${ }^{1}$ Laboratory of Information Processing and Telecommunications (LTIT), Department of Electrical \\ Engineering, Faculty of Technology, Tahri Mohamed University, Algeria \\ ${ }^{2}$ Biomedical Engineering Laboratory, Biomedical Engineering Department, Faculty of Technology, \\ Abou Bekr Belkaïd University, Algeria
}

\begin{tabular}{|c|c|}
\hline Article Info & ABSTRACT \\
\hline Article history: & Ultrasound Doppler signal processing is a necessity in several disciplines of \\
\hline Received Nov 20, 2018 & $\begin{array}{l}\text { medicine. The interpretation of these signals allows a good diagnosis of } \\
\text { patients in order to detect certain diseases. In this work, we will use different }\end{array}$ \\
\hline Revised Jan 21, 2019 & non-parametric time-frequency techniques such as the Wigner-Ville \\
\hline Accepted Feb 28, 2019 & $\begin{array}{l}\text { distribution (WVD), the Pseudo-Wigner-Ville distribution (PWVD), } \\
\text { the smoothed pseudo Wigner-Ville distribution }(S P W V D) \text {, and the S-transform }\end{array}$ \\
\hline Keywords: & $\begin{array}{l}\text { in order to analyze the Ultrasonic Doppler signal of the } \\
\text { femoral arteries. }\end{array}$ \\
\hline
\end{tabular}

Pseudo-Wigner-Ville

distribution

Smoothed pseudo Wigner-Ville

distribution

S-transform

Ultrasound Doppler signal

Copyright (C) 2019 Institute of Advanced Engineering and Science.

Wigner-Ville distribution All rights reserved.

\section{Corresponding Author:}

Latfaoui Mahieddine,

Laboratory of Information Processing and Telecommunications (LTIT),

Department of Electrical Engineering,

Faculty of Technology,

Tahri Mohamed University, Bechar, Algeria.

Email: m.latfaoui@gmail.com

\section{INTRODUCTION}

Spectral analysis of ultrasound Doppler signals is an important tool in the diagnosis of vascular diseases and in the study of stenosis. The most widely used method to analyze a Doppler signal in real time is the STFT short-time Fourier transform. Unfortunately, this method has a major disadvantage, which is the absence of a compromise between the resolution of the frequency and the resolution time. However, a good time and frequency resolution is necessary to characterize the non-stationarity of the signal. In addition, it must be assumed that the signal can be processed as a stationary stochastic process in narrower analysis windows.

The basic idea of time-frequency analysis is to understand and describe situations where the frequency content of a signal changes over time. Although the time-frequency analysis is almost 70 years old, significant progress has been made over the last 20 years. In particular, time-frequency representation has received considerable attention as a powerful tool of high resolution and accuracy for the analysis of a variety of biomedical signals such as ultrasound Doppler signals, as well as signals from other fields [1], [2].

In this paper, we have limited our study to a few distributions such as the Wigner-Ville distribution $(W V D)$, the Pseudo Wigner-Ville-distribution $(P W V D)$, and the smoothed pseudo Wigner-Ville distribution $(S P W V D)$. Other techniques based on the wavelet transform such as the S-transform can also be applied to 
analyze the Doppler blood flow signal. The $W V D, P W V D$ and $S P W V D$ distributions were introduced for non stationary spectral analysis and showed good time-frequency resolution [3].

\section{METHODS OF ANALYSIS}

\subsection{The Fourier Transform}

Ultrasound Doppler signals are non-stationary signals, because the frequency that represents the speed of red blood cells varies with time, in addition Doppler signals is frequency-modulated by the speed of red blood cells movement.

To perform a spectral analysis of a Doppler signal, we use the Fourier transform given by the following relation:

$$
X(f)=\int_{-\infty}^{+\infty} x(t) e^{-j 2 \pi f t} d t
$$

This transform provides information on the frequency components of the signal studied; their amplitude and their phase. The major disadvantage of this application is that it causes a loss of information concerning their temporal location [3]-[5].

\subsection{The S-Transform}

The S-transform of a signal $x(t)$ representing a time-frequency analysis $x(t)$ was developed by Stokwell in 1996. This transform is similar to the short-term Fourier transform, except that the amplitude and the width of the analysis window is variable depending on the frequency, as is the case in wavelet analysis; this transform is given by:

$$
S(\tau, f)=\int_{-\infty}^{+\infty} x(t) w(t-\tau, f) \cdot e^{-j 2 \pi f t} d t
$$

Where the mother wavelet is written by:

$$
w(t, f)=\frac{|f|}{k \sqrt{2 \pi}} e^{\frac{-f^{2} t^{2}}{2 k^{2}}}, k>0
$$

Where $t$ is a time variable, $f$ is the frequency, and $k$ is a scale factor that controls the number of oscillations in the mother wavelet [6].

\subsection{The discrete $\mathbf{S}$ Transform}

Let us consider $X[k T], k=0 ; 1 ; \ldots ; N-1$ a discrete signal, corresponding to $x(t)$ with a sampling period $T$. The discrete Fourier transform is given by [7]:

$$
X\left[\frac{\mathrm{n}}{\mathrm{NT}}\right]=\frac{1}{\mathrm{~N}} \sum_{\mathrm{k}=0}^{\mathrm{N}-1} \mathrm{x}[\mathrm{kT}] \mathrm{e}^{-\frac{\mathrm{j} 2 \pi \mathrm{nk}}{\mathrm{N}}}
$$

Where $n=0,1 \ldots, N-1$. In the discrete case, the $\mathrm{S}$ transform is the projection of the vector defined by $x[k T]$ in a non-orthogonal basis of vectors and the elements of the $\mathrm{S}$ transform are not independent. Each basis vector (of the Fourier transform) is divided into $N$ localized vectors by an element-by-element product with the shifted Gaussians, such that the sum of these $N$ localized vectors is equal to the original basis vector. By using the discrete version of the (2), and when placing $f \rightarrow \frac{n}{N T}$ and $\tau \rightarrow i T$, the $\mathrm{S}$ transform of a discrete signal $x[k T]$ is given by:

$$
\mathrm{S}\left[\mathrm{iT}, \frac{\mathrm{n}}{\mathrm{NT}}\right]=\sum_{\mathrm{m}=0}^{\mathrm{N}-1} X\left[\frac{\mathrm{m}+\mathrm{n}}{\mathrm{NT}}\right] \mathrm{e}^{-\frac{2 \pi^{2} \mathrm{~m}^{2}}{\mathrm{n}^{2}}} \mathrm{e}^{\mathrm{j} 2 \pi \mathrm{mi}}
$$

and for the $n=0$ voice, is equal to the constant defined by:

$$
\mathrm{S}[\mathrm{iT}, 0]=\frac{1}{\mathrm{~N}} \sum_{\mathrm{m}=0}^{\mathrm{N}-1} \mathrm{X}\left[\frac{\mathrm{m}}{\mathrm{NT}}\right]
$$

Where $i, m$ and $n=0,1 \ldots, N-1$.

The (6) puts the constant average of the signal in the way corresponding to zero frequency. Thus, the existence of the reverse is ensured in the general case. The discrete $\mathrm{S}$ transform faces the problems 
of sampling and finite length, causing the implicit periodicity in the time and frequency fields. The convolution operations are implicitly ' wrap-around ' causing edge effects. The reverse of the discrete $S$ transform is given by:

$$
\mathrm{x}[\mathrm{kT}]=\frac{1}{\mathrm{~N}} \sum_{\mathrm{n}=0}^{\mathrm{N}-1}\left\{\sum_{\mathrm{i}=0}^{\mathrm{N}-1} \mathrm{~S}\left[\frac{\mathrm{n}}{\mathrm{NT}}, \mathrm{iT}\right]\right\} \mathrm{e}^{\frac{\mathrm{i} 2 \pi \mathrm{k}}{\mathrm{N}}}
$$

\subsection{The Analytical Signal}

The analytical signal is used to avoid the overlap of the frequency components in the time-frequency plane; this signal is obtained by applying the Hilbert transform:

$$
x_{z}(t)=x(t)+j H\{x(t)\}
$$

Where $H\{x(t)\}$ represents the Hilbert transform of $x(t)$

$$
H\{x(t)\}=\frac{1}{\pi} \operatorname{vp} \int_{-\infty}^{+\infty} \frac{x(\tau)}{t-\tau} \cdot d \tau
$$

$v p$ being the abbreviation of Cauchy's main value [8].

\subsection{The Wigner-Ville Transform}

This approach was developed by Wigner for use in physics, but was later applied by Ville for signal processing. This Wigner-Ville transform provides time-frequency decomposition without any restriction on temporal and frequency resolutions. It appears to be perfectly suited to the analysis of non-stationary signals since it does not require assumptions about the signal itself. $T W V$ is defined by:

$$
W V_{x}(t, f)=\int_{-\infty}^{+\infty} x\left(t+\frac{\tau}{2}\right) x^{*}\left(t-\frac{\tau}{2}\right) e^{-j 2 \pi f \tau} d \tau
$$

This formula represents the energy of a signal $x$ at time $t$ and at frequency $f$. Unfortunately, the nonlinearity of this transform has disastrous consequences manifested by the appearance of interference and negative energies in the time-frequency distribution of the signal energy. These phenomena are remarkable when there are two distinct events in time and frequency [8]. This phenomenon is unrealistic from a physical point of view, and suggests difficulties in the interpretation of the $T W V$. Therefore, and in order to extract reliable results, it is necessary to avoid these negative energies by post-treatment that will affect the resolution properties of the transform. The $D W V$ is related to the ambiguity function $A_{x}(n, z)$, which is a time-frequency correlation measurement; by a two-dimensional Fourier transform relation:

$$
A_{x}(n, \tau)=\int_{-\infty}^{+\infty} \int_{-\infty}^{+\infty} x\left(u+\frac{\tau}{2}\right) x^{*}\left(u-\frac{\tau}{2}\right) e^{j 2 \pi n u} d u=\int_{-\infty}^{+\infty} W_{x}(t, f) e^{j 2 \pi(n t+\tau f)} d t \cdot d f
$$

This makes it possible to consider the classical problems involving the ambiguity function (in particular optimal detection-estimation, adapted filtering and signal synthesis) in a dual way by working directly on the time-frequency plane with the VWD.

\subsection{The Pseudo Wigner-Ville Transform}

The pseudo Wigner-Ville Distribution $(P W V D)$ is a short-term Wigner-Ville transform. An observation time window $\mathrm{h}$ is introduced, and this distribution smooths out in the frequency directions only where the interference will not be attenuated in time.

$$
\operatorname{PWV}_{x}(t, f)=\int_{-\infty}^{+\infty} h(\tau) x\left(t+\frac{\tau}{2}\right) x^{*}\left(t-\frac{\tau}{2}\right) e^{-j 2 \pi f \tau} d \tau
$$

If we introduce a temporal smoothing in the equation using a frequency window $g$, we will have a smoothed pseudo Wigner-Ville distribution [9], [10].

\subsection{The Smoothed Pseudo-Wigner-Ville Transform}

The pseudo Wigner-Ville distribution has the effect of reducing the small parasitic ripples in the frequency direction. However, the parasitic ripples appearing in the $P W V D$ can be further reduced by smoothing the $P W V D$, this time in the direction of the time of the plan $(t, f)$, where we have a new transform, i.e. that of the smoothed pseudo-Wigner-Ville distribution given by the following relation [11], [12]: 


$$
\operatorname{SPWV}_{x}(t, f)=\int_{-\infty}^{+\infty} h(\tau) \int_{-\infty}^{+\infty} g(s-t) x\left(s+\frac{\tau}{2}\right) x^{*}\left(s-\frac{\tau}{2}\right) \cdot d s \cdot e^{-j 2 \pi f \tau} d \tau
$$

\section{RESULTS AND DISCUSSION}

The major theoretical advantage of the methods studied in the previous part lies in their ability to define more precisely the non-stationary signals in the time-frequency space without any hypothesis on the characteristics of the signal. Spectral analysis of non-stationary signals requires the implementation of specific tools to describe a temporal evolution of frequency characteristics. In addition, they can provide precise information on the speed of blood flow in the femoral arteries [12].

The Doppler blood flow signal is a non-stationary Gaussian random signal with a relatively slow average variable frequency. The work of Keeton and S. Kara shows that the Ultrasonic Doppler signal of the femoral arteries can be considered as a stationary signal on segments from $12 \mathrm{~ms}$ to $20 \mathrm{~ms}$; the choices of the size of the analysis windows is based on this proposition [13].

The ultrasound Doppler signals studied in this work are real signals from St Marie's Hospital in Leicester, and have a sampling frequency of $10240 \mathrm{HZ}$, and duration of $4 \mathrm{~s}$. The following Figure 1 shows the time representation of one of these signals.

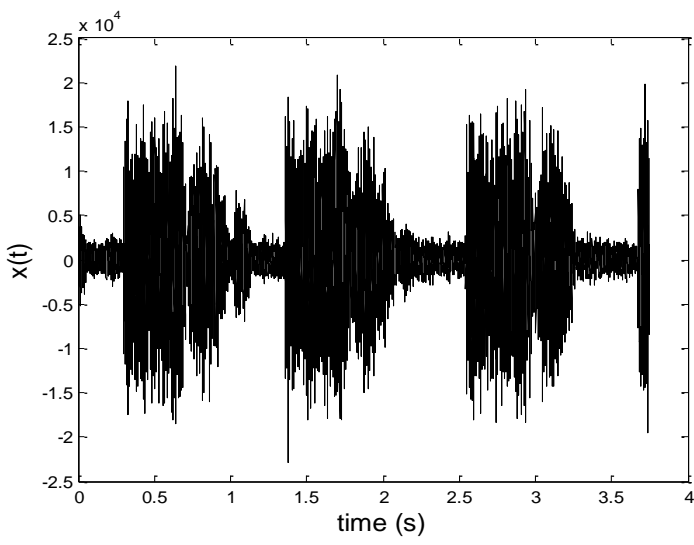

(a)

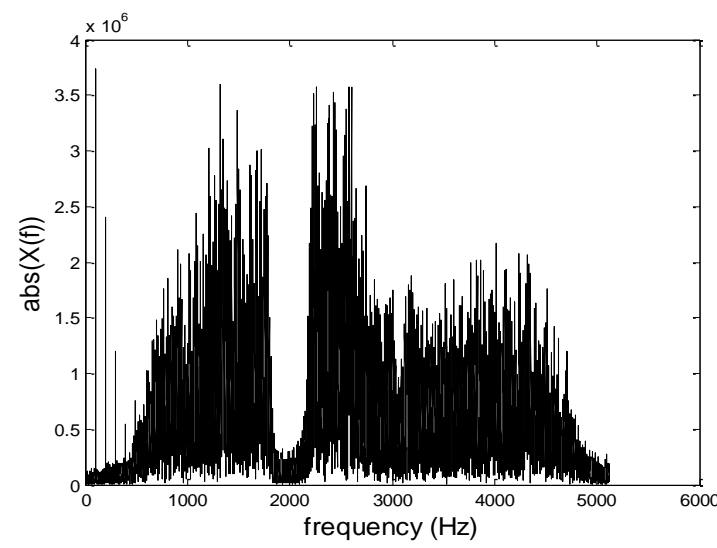

(b)

Figure 1. (a) Temporal representation, (b) Spectrum of doppler ultrasound signal

A time-frequency distribution is used to analyze and process non-stationary signals in the timefrequency domain, as well as to improve the performance of time-frequency analysis with respect to the spectral analysis of biomedical signals.

Figures (2a) and (2b) represent the sonograms obtained by $P W D$ and $S P W D$ of the analytical Doppler signal, using Hamming windows of different widths. Note, however, that small parasitic ripples are much smaller in $P W V D s$ than in WVDs. It can be seen that the sonograms obtained by the $P W D$ and $S P W D$ have a better frequency resolution than the results obtained by $W V D$. The degraded frequency resolution is the disadvantage of the $P W V D$ compared to the $W V D$, but the $P W V D$ always keeps the same temporal resolution, since the convolution operation only acts on the frequency direction. This is interesting compared to the spectrogram, which provides a representation in which the frequency and time resolutions are always related. A judicious choice of types and widths of smoothing windows is a necessary condition for having a good resolution on the time-frequency plan in the application of $P W V D$ or $S P W V D$ on ultrasonic Doppler signals [14]-[16].

The $W V D$ retains the temporal and frequency supports of a signal, and thus allows a precise location both in time and in frequency. This is another fundamental property that enables to conveniently separate the different events on the time-frequency plane. 


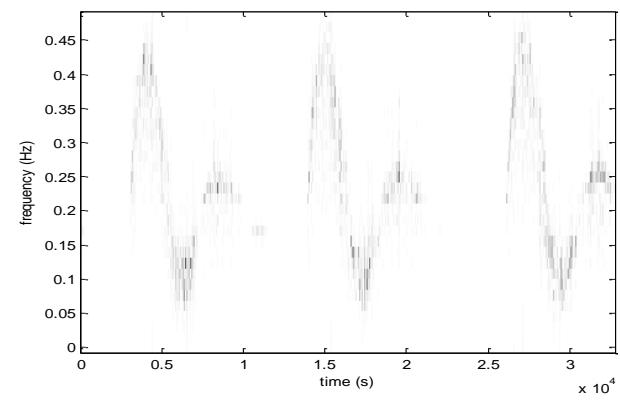

(a)

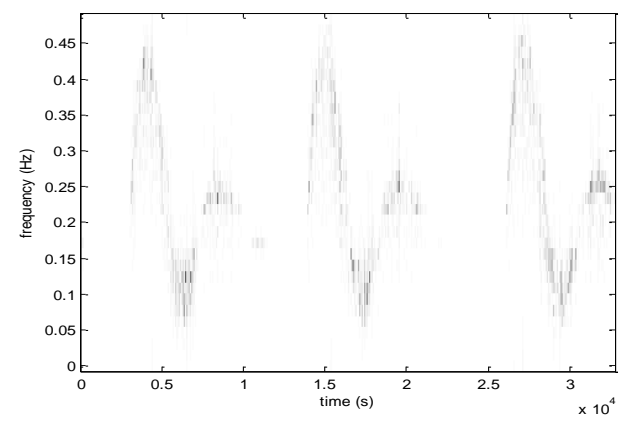

(c)

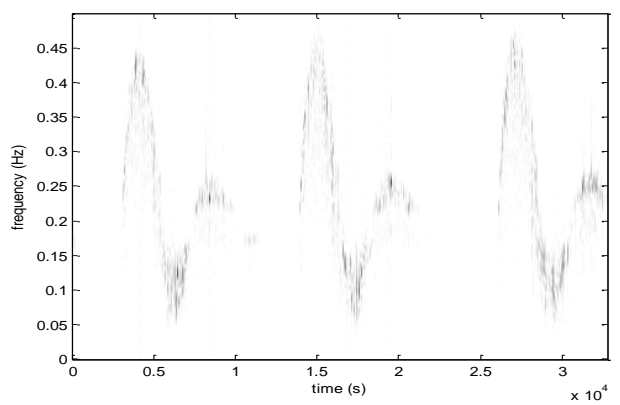

(b)

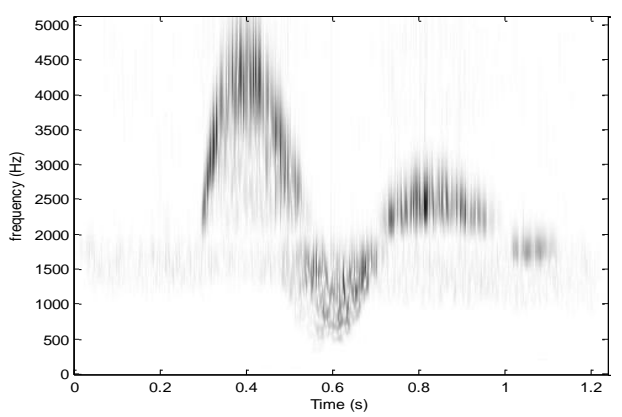

(d)

Figure 2. Femoral arterial doppler sonograms using: (a) WVD, (b) PWVD, (c) SPWVD, (d) S-transform

Consequently, due its bilinear structure, the WVD naturally creates interference terms between the different components of a signal. These terms, which are in some ways useful because they carry information on the relationships between the different components, are generally disruptive vis-à-vis the readability of a time-frequency diagram. It can indeed be shown that they are characterized by an oscillatory structure, in contrast with the term "signal" with softer behavior [17], [18].

The sonograms obtained by the SPWVD show in Figure 2(c) give better performances compared to other time-frequency techniques. This technique has a better resolution and a minimum of interference and spectral spread compared to the other techniques due to the Wigner-Ville distribution.

Figure 2(d) illustrates the sonogram obtained by the S-transform; this result shows a quantitative improvement in the time-frequency analysis compared to the results obtained by Wigner-Ville distributions, and this is due to the use of wavelets in this technique [19], [20].

Note, however, that small parasitic ripples are much smaller in TPWVs than in TWVs. This aspect of compromise between the frequency resolution and the degree of attenuation of the parasites (interference terms) is in fact a typical and classic problem that is encountered in most practical situations (multi-component signals). This is also the reason why the use of the TPWV always requires a judicious choice of the window (in terms of type and width) according to the characteristics of the signal processed and the information sought [21], [22].

\section{SBI CALCULATION}

The frequency envelopes that are extracted from the sonograms obtained by different methods will be applied to the input of an order 6 averaging filter to obtain a better resolution at the systolic peaks [13].

The degree of stenosis (given in percentage \%) is expressed as the ratio of the diameter reduced by the stenosis to the actual diameter of the artery. Maximum and mean frequency envelopes of the carotid arterial extracted from: (a) WVD, (b) PWVD, (c) SPWVD, (d) S-transform as shown in Figure 3.

The flow upstream of the stenosis is laminar and the red blood cells flow with a speed called average speed " $V_{\text {moy }}$ ". At the level of the stenosis, the speed of the red blood cells increases because of the arterial narrowing, to maintain a constant flow. In this case, the speed is maximal, and is called " $V_{\max }$ ". The effect of stenosis on the flow of blood in the arteries as shown in Figure 4. 


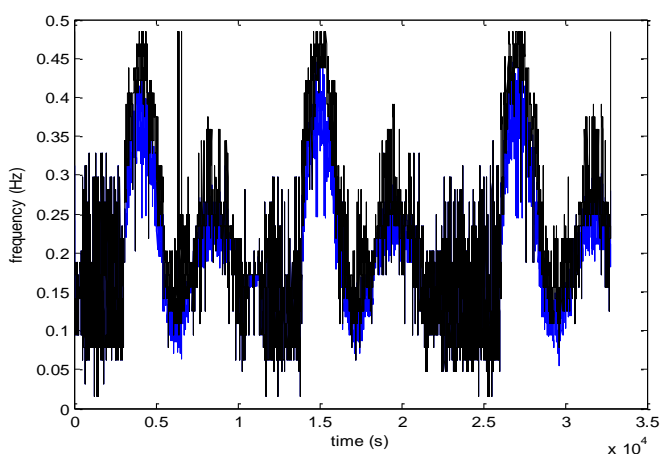

(a)

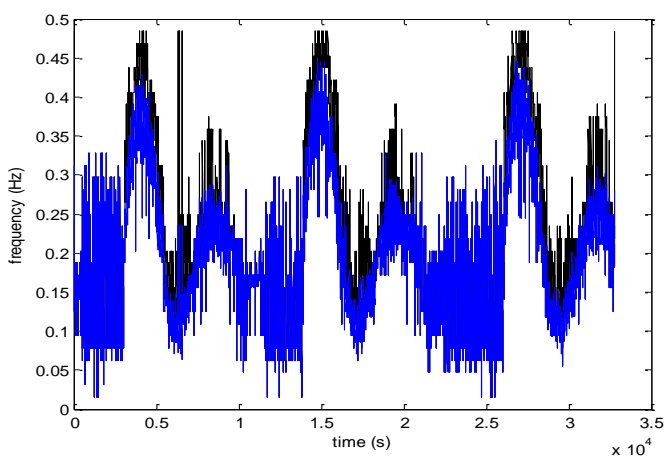

(c)

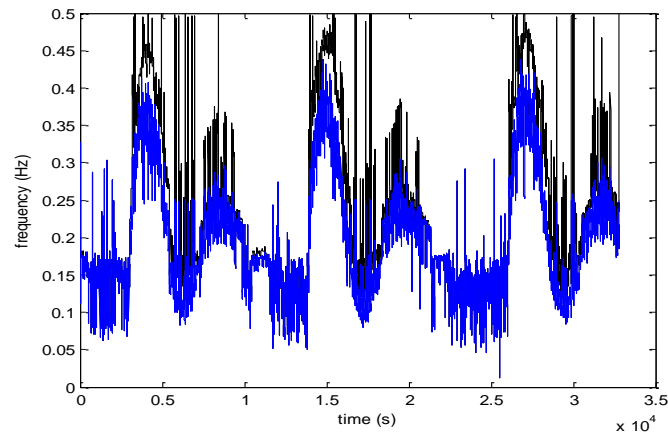

(b)

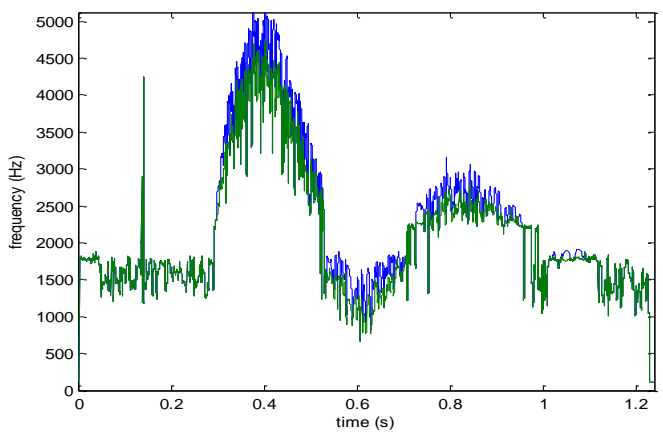

(d)

Figure 3. Maximum and mean frequency envelopes of the carotid arterial extracted from: (a) WVD, (b) $P W V D$, (c) $S P W V D$, (d) S-transform

Immediately downstream of the stenosis, when the diameter increases sharply, a swirling motion appears and totally disorganizes the flow; this allows the red blood cells to take multiple speeds and in all directions.

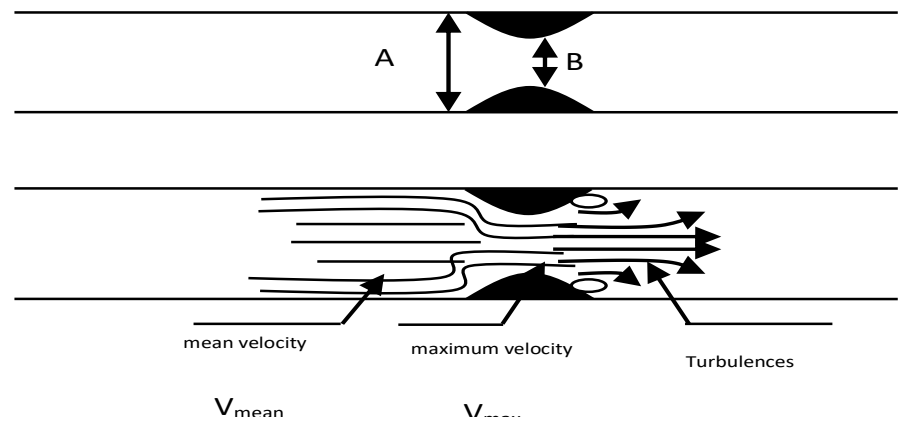

Figure 4. The effect of stenosis on the flow of blood in the arteries

The average value of these speeds gives the average speed of the flow Vmean. All velocities measured at the stenosisand near it, including the maximum velocity " $V_{\max }$ ", are represented by the Doppler spectrum as frequency components. In this case, we can say that the maximum frequency of the Doppler spectrum " $f_{\text {max }}$ " measures the maximum speed of the flow " $V_{\text {max }}$ ", and the average frequency " $f_{\text {mean }}$ " measures the average speed "V $V_{\text {mean }}$.

$f_{\max }$ is calculated by the average of the frequencies at the systolic peaks. The results obtained are presented as follows:

Time frequency analysis of ultrasound doppler signal by S-transform and... (M. Latfaoui) 
Relationship between $S B I$ and the degree of stenosis:

$$
S B I=\frac{f_{\max }-f_{\operatorname{mean}}}{f_{\max }}
$$

Increasing the degree of stenosis increases the flow rateat the level of arterial narrowing; this is interpreted by the following equation:

$$
B=A \cdot\left(\frac{V_{\text {mean }}}{V_{\max }}\right)
$$

Where Vmean is the flow velocity when the diameter of the artery is equal to $A$, and $V_{\text {max }}$ becomes the flow velocity when the diameter is equal to $B$.

Then express the degree of stenosis can be expressed as a function of Vmean and Vmax:

$$
\text { Degree of stenosis }=\frac{(A-B)}{A} \times 100 \%=\frac{\left(V_{\max }-V_{\text {mean }}\right)}{V_{\max }} \times 100 \%
$$

Since the velocities $V_{\text {max }}$ and $V_{\text {mean }}$ respectively represent the frequencies $f_{\text {max }}$ and $f_{\text {mean }}$ of the Doppler spectrum, we can express the degree of stenosis as a function of $f_{\text {max }}$ and $f_{\text {mean }}$ :

$$
\text { Degree of stenosis }=\frac{\left(f_{\max }-f_{\operatorname{mean}}\right)}{f_{\max }}=S B I
$$

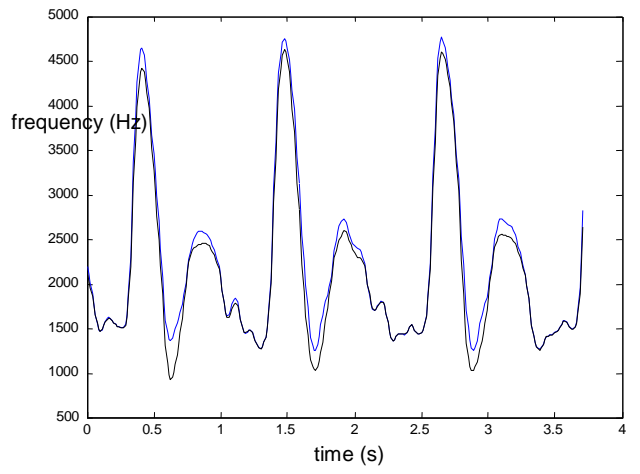

(a)

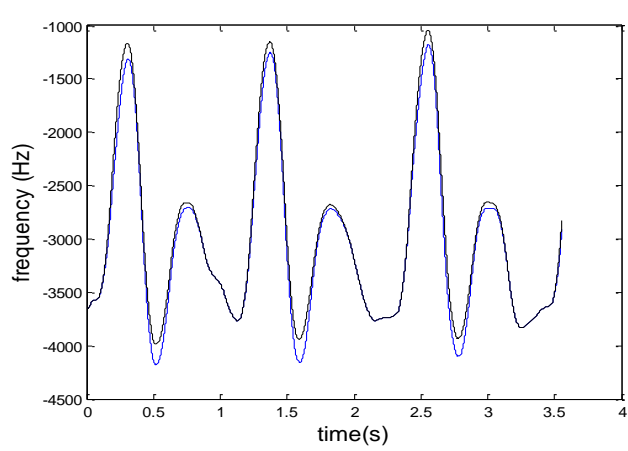

(c)

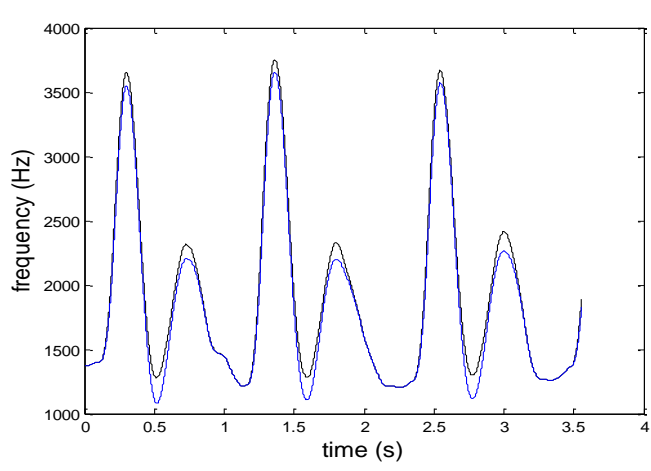

(b)

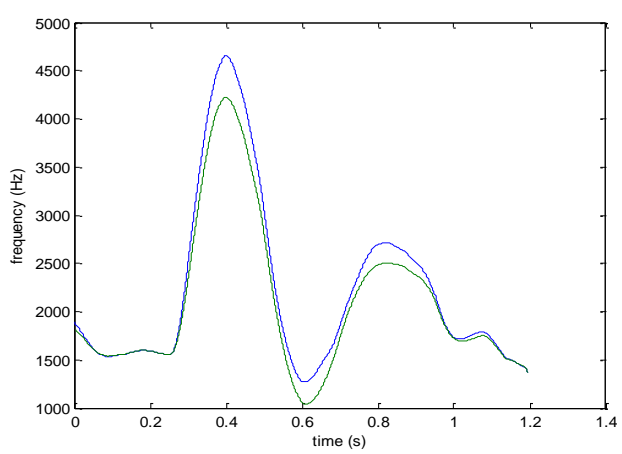

(d)

Figure 5. Filtered envelopes fmax and fmean of the artery femoral sonograms using: (a) WVD, (b) PWVD, (c) SPWVD, (d) S-transform

This last equation measures the spectral broadening between $f_{\text {max }}$ and $f_{\text {mean }}$ with respect to $f_{\text {max }}$; which represents the spectral broadening index $S B I$.

The frequencies $f_{\text {max }}$ and $f_{\text {mean }}$ are obtained respectively by calculating the average of the different $f_{\max }$ and $f_{\text {mean }}$ on a succession of systolic cycles generated by the sonograms. 


\section{CONCLUSION}

The main objective of this work is to present the performances of several non-parametric timefrequency techniques, namely the distributions of Wigner-Ville, pseudo Wigner-ville, smoothed pseudo Wigner Ville and the S-transform in the analysis of Doppler ultrasound signals. The application of these methods on several Doppler signal recordings of normal Doppler arteries or showing certain pathologies allows us to obtain sonograms to have a better time-frequency analysis. Based on the results obtained by the different distributions of Wigner-Ville and the S-transform, it is clear that the sonograms have been reached by the WVD, but that the use of the SPWD makes it possible to smooth these interferences; the S-transform generates sonograms whose spectral and temporal resolutions are better compared to the Wigner-Ville distributions. Our study is followed by a quantified assessment of the severity of the SBI study. The SBI was calculated for different normal and pathological cases. Results have showen that there is a relationship between $S B I$ and the degree of surface stability.

\section{REFERENCES}

[1] Jianping M, Jin J., "Analysis and design of modified window shapes for S-transform to improve time-frequency localization," Mechanical Systems and Signal Processing, vol. 58, pp. 271-284, 2015.

[2] D.Urynbassarova, B. Zhao Li, R.Tao, "Convolution and Correlation Theorems for Wigner-Ville Distribution Associated with the Offset Linear Canonical Transform," Optik- International Journal for Light and Electron Optics, vol. 157, pp. 455-466, 2018.

[3] F. Parsia George, et al., "Recognition of emotional states using EEG signals based on time-frequency analysis and SVM classifier," Inter. Journal of Electrical and Computer Engineering (IJECE), vol. 9, pp.1012-1020, 2019.

[4] A. A. Adly, S.K. Abd-El-Hafiz., "A Deconvolution Approach to the Three Dimensional Identification of Cracks in Magnetic Slabs, " Inter. Journal of Electrical and Computer Engineering (IJECE), vol. 7, pp. 2357-2364, 2017.

[5] W. Prasetyo, M. Alaydrus., "The condition monitoring of diesel engines using acoustic signal analysis," Indonesian Journal of Electrical Engineering and Computer Science (IJEECS), vol. 13, pp. 179-185, 2019.

[6] Z. Zhang, Y. Sun, K. Berteussen, "Time Frequency Wavenumber Analysis of Surface Waves and Signal Enhancement Using S-transform," Journal of Computational Acoustics, vol. 23, pp. 1-13, 2015.

[7] HK. Vydana, AK. Vuppala, "Detection of fricatives using S-transform," The Journal of the Acoustical Society of America, vol. 140 (5), pp. 3895-3907, 2016.

[8] G. Andria, M. Savino, "Application of Wigner-Ville Distribution to Measurements on Transient Signals," IEEE Transactions on Instrumentation and Measurements, vol. 43(2), pp. 187-193, 1994

[9] M. Szmajda, K. Górecki, J. Mroczka, "Gabor Transform, SPWVD, Gabor-Wigner Transform And Wavelet Transform Tools For Power Quality Monitoring," Metr. and Measurement Systems, vol. 17 (3), pp. 383-396, 2010.

[10] S. Pikula, P. Beneš, "A New Method for Interference Reduction in the Smoothed Pseudo Wigner-Ville Distribution," Proceedings of the 8th Int. Conference on Sensing Technology. Liverpool, UK, 2014, pp. 599-603.

[11] C. Yen Mei, A.Z. Sha'ameri, "Adaptive windowed cross Wigner-Ville distribution as an optimum phase estimator for PSK signals," Digital Signal Processing, vol. 23(1), pp. 289-301, 2013.

[12] P.I.J. Keeton, F.S. Schlindwein, "Spectral broadening of clinical Doppler signals using FFT and autoregressive modelling," European Journal of Ultrasound 7, vol. 7(3), pp. 209-218, 1998.

[13] F.S. Schlindwein, D.H. Evans, "Selection of order of autoregressive model for spectral analysis of Doppler ultrasound signals," Ultrasound Med Biol., vol. 16(1), pp. 81-91, 1990.

[14] Song YuE, et al., "The Wigner-Ville Distribution Based on the Linear Canonical Transform and Its Applications for QFM Signal Parameters Estimation," Journal of Applied Mathematics, vol. 2014, pp. 1-14, 2014.

[15] M. Latfaoui, F. Bereksi Reguig, "Packets Wavelets and Stockwell Transform Analysis of Femoral Doppler Ultrasound Signals," Inter. Journal of Electrical and Computer Engineering (IJECE), vol. 8, pp. 4212-4220, 2018.

[16] S.Kara, "Classification of mitral stenosis from Doppler signals using short time Fourier transform and artificial neural networls," Expert Systems witn applications, vol. 33, pp. 468-475, 2007.

[17] J. Wu, Z.Ma, Y. Zhang, "A Time-Frequency Research for Ultrasonic Guided Wave Generated from the Debonding Based on a Novel Time-Frequency Analysis Technique," Shock and Vibration, vol. 2017, pp. 1-11, 2017.

[18] X. Hua, J. Liu, "A Novel Fast Algorithm for the Pseudo Winger-Ville Distribution," Journal of Communications Technology and Electronics, vol. 60(11), pp. 1238-1247, 2015.

[19] Y. Wang, et al., "Analysis of micro-Doppler signatures of vibration targets using EMD and SPWVD," Neurocomputing, vol. 171, pp. 48-56, 2016.

[20] S. Dhanya, V.S. Kumari Roshni, "Comparison of various texture classification methods using multiresolution analysis and linear regression modelling," SpringerPlus, vol. 5(1), pp. 1-18, 2016.

[21] A. Moukadem, et al., "A new optimized Stockwell transform applied on synthetic and real non-stationary signals," Digital Signal Processing, vol. 46, pp. 226-238, 2015.

[22] S. Li X, et al. "Analysis and Simplification of Three-Dimensional Space Vector PWM for Three-Phase Four-Leg Inverters," IEEE Transactions on Industrial Electronics, vol. 58, pp. 450-464, 2011. 